\title{
Genome-wide identification, characterization, and expression analysis of the $M L O$ gene family in Cucumis sativus
}

\author{
S.J. Zhou ${ }^{1}$, Z. Jing ${ }^{2}$ and J.L. Shi ${ }^{2}$ \\ ${ }^{1}$ Institute of Vegetables, Zhejiang Academy of Agricultural Sciences, \\ Hangzhou, Zhejiang Province, China \\ ${ }^{2}$ Southern Zhejiang Key Laboratory of Crop Breeding, \\ Department of Agriculture and Biotechnology, \\ Wenzhou Vocational College of Science and Technology, Wenzhou, China \\ Corresponding author: S.J. Zhou \\ E-mail: sjzhou_zj@sina.com
}

Genet. Mol. Res. 12 (4): 6565-6578 (2013)

Received November 14, 2012

Accepted September 25, 2013

Published December 11, 2013

DOI http://dx.doi.org/10.4238/2013.December.11.8

\begin{abstract}
Mildew resistance locus o (MLO) is a plant-specific seven-transmembrane (TM) gene family. Several studies have revealed that certain members of the $M L O$ gene family mediate powdery mildew susceptibility in three plant species, namely, Arabidopsis, barley, and tomato. The sequenced cucumber genome provides an opportunity to conduct a comprehensive overview of the $M L O$ gene family. Fourteen genes (designated CsMLO01 through CsMLO14) have been identified within the Cucumis sativus genome by using an in silico cloning method with the MLO amino acid sequences of Arabidopsis thaliana and rice as probes. Sequence alignment revealed that numerous features of the gene family, such as TMs, a calmodulin-binding domain, peptide domains I and II, and 30 important amino acid residues for MLO function, are well conserved. Phylogenetic analysis of the $M L O$ genes from cucumber and other plant species reveals seven different clades (I through VII). Three of these clades comprised $M L O$ genes from $A$.
\end{abstract}


thaliana, rice, maize, and cucumber, suggesting that these genes may have evolved after the divergence of monocots and dicots. In silico mapping showed that these CsMLOs were located on chromosomes $1,2,3,4,5$, and 6 without any obvious clustering, except CsMLO01. To our knowledge, this paper is the first comprehensive report on $M L O$ genes in $C$. sativus. These findings will facilitate the functional characterization of the $M L O s$ related to powdery mildew susceptibility and assist in the development of disease resistance in cucumber.

Key words: MLO; Cucumber; Bioinformatics; Powdery mildew

\section{INTRODUCTION}

Mildew resistance locus o (MLO) proteins belong to a unique plant-based family of seven-transmembrane domain (TM) proteins that contain a C-terminal calmodulin-binding domain (CaMBD) and an extracellularly located N-terminus (Devoto et al., 1999; Kim et al., 2002a). Recessive mutations in the $M L O$ gene confer durable broad-spectrum resistance to all known isolates of the barley powdery mildew (PM) fungus Blumeria graminis f. sp hordei (Bgh) (Buschges et al., 1997). Naturally occurring broad-spectrum resistance to Bgh was first observed in Ethiopian barley landraces (Jørgensen, 1992; Piffanelli et al., 2004).

Although $M L O$ genes were first described in grasses, some members of this family are also inferred to play a role in modulating host response to the phytopathogenic PM fungus in dicots. For example, in Arabidopsis, the AtMLO2 gene shows significantly reduced susceptibility to Golovinomyces orontii. Two other closely related Arabidopsis genes, AtMLO6 and AtMLO12, are also mutated, along with $A t M L O 2$, to achieve complete PM resistance (Consonni et al., 2006). Subsequently, the absence of $M L O$ genes (SlMLO) was demonstrated to be the cause of PM resistance in tomato (Bai et al., 2008). Recently, Pavan et al. (2011) demonstrated that pea PM er1 resistance is associated with loss-of-function mutations at an $M L O$-homologous locus. A biologically active $M L O$ may be a general requirement for PM pathogenesis in both monocotyledonous and dicotyledonous plants.

The interaction between $M L O$ genes and PM may have a special mechanism. Nevertheless, knowledge of the precise action mechanism of MLO proteins is limited. The function of $M L O$ genes from plants and bacteria in the signal transduction process may be identified for determining the precise MLO mechanism. MLOs are calmodulin-binding proteins, and calmodulin binding promotes the susceptibility of barley to PM (Kim et al., 2002b). Moreover, pharmacological studies suggest that the influx of $\mathrm{Ca}^{2+}$ ions is important for this MLO function (Kim et al., 2002a). Therefore, $\mathrm{Ca}^{2+}$ may be a candidate signal because plant cells generate a transient $\mathrm{Ca}^{2+}$ signal in response to pathogen attack (Jabs et al., 1997; Xu and Heath, 1998). According to a previous report, two genes, $R O R 1$ and $R O R 2$, are required for complete $M L O$ mediated resistance (Freialdenhoven et al., 1996; Peterhansel et al., 1997), whereas $M L O$ mediated defense suppression might likely involve one or several small GTP-binding proteins of the ROP family (Schultheiss et al., 2002). Consequently, Bhat et al. (2005) suggested that MLO, ROR2, and, potentially, additional proteins might form a novel pathogen-triggered microdomain at biotic stress sites.

Cucumber PM [Sphaerotheca fuliginea (Schlecht.) Poll] is a major disease that affects cucumber yield and quality. Several genes resistant to PM have been identified, namely, Pm-1, 
$P m-2, P m-3, P m-4$, and $P m-H$. The involvement of more than one gene in PM resistance in several cucumber accessions has been reported in several studies (Pierce and Wehner, 1990). Several linkages between the PM resistance locus and markers have also been reported (Fanourakis and Simon, 1987; Walters et al., 2001; Zhang et al., 2007). Moreover, a correlation exists between the presence of genes and resistance to PM as well as downy mildew (DM) (Van Vliet and Meijsing, 1977; Pivovarov, 1988). Linkage analysis studies indicate that the DM and PM genes are located either at the same locus or in closely linked loci (Fanourakis, 1984). The first quantitative trait loci (QTLs) for PM resistance in cucumber were identified by Sakata et al. (2006) who mapped 5 QTLs in four linkage groups on an unsaturated linkage map.

Apart from the reports of barley and tomato $M L O s$ in PM pathogenesis, only a few references were available for other $M L O$ genes. Thus, an in-depth analysis of the whole MLO family in plants is necessary. In 2009, the cucumber genome was sequenced by Chinese researchers working on the "Chinese Long" inbred line 9930 (Huang et al., 2009). The resulting genome sequence provides an opportunity to conduct a comprehensive overview of the $M L O$ gene family in this species. We identified $14 M L O$ genes in the cucumber genome in this study, analyzed their structural features, assessed the phylogenetic relationship between MLOs from cucumber and other plants, and performed in silico mapping of the $M L O$ genes on the chromosomes. The role of epigenetics in the evolution of these $M L O$ genes has also been discussed.

\section{MATERIAL AND METHODS}

\section{Sequence database search and domain detection}

MLO protein sequences in Cucumis sativus were identified through MLO sequences from other plant species by using the Basic Local Alignment Search Tool (BLAST) of the Cucumber Genome Browser (http://cucumber.genomics.org.cn/page/cucumber/index.jsp) provided by Institute of Vegetables and Flowers, Chinese Academy of Agricultural Sciences (Huang et al., 2009). The query sequences were from previously published data on Arabidopsis thaliana and Oryza sativa (Devoto et al., 2003; Liu and Zhu, 2008). In addition, select $M L O$ genes from barley (Hordeum vulgare), maize (Zea mays), wheat (Triticum aestivum), pea (Pisum sativum), and tomato (Solanum lycopersicum) were also included to understand the phylogenetic relationship of $M L O$ genes in plants. Sequence of MLO proteins from these plant species was downloaded from various databases. A. thaliana MLO proteins reported by Devoto et al. (2003) were retrieved from The Arabidopsis Information Resource (TAIR) (http://www.arabidopsis.org/browse/genefamily/mlo.jsp). The sequences of the rice MLO proteins reported by Liu and Zhu (2008) were retrieved from the National Center for Biotechnology Information (NCBI) (http://www.ncbi.nlm.nih.gov/). Select MLO protein sequences from the remaining five plant species were retrieved from the GenBank database. Finally, all the MLO protein sequences were submitted to the PFAM database to verify the presence of the MLO domain.

\section{Multiple sequence alignment and phylogenetic tree construction}

Multiple alignments of full amino acid sequences of cucumber MLOs were performed using the CLUSTAL_X feature in the BioEdit software (Thompson et al., 1997). The phylo- 
genetic tree was constructed by aligning all the MLO protein sequences of tomato (Bai et al., 2008) and Arabidopsis (Devoto et al., 2003) and select proteins from barley, maize, wheat, pea, and tomato with the ClustalX Version 1.83 program by using the neighbor-joining algorithm implemented in the Molecular Evolutionary Genetics Analysis (MEGA) software, version 5.0 (Tamura et al., 2011). Bootstrapping (1000 replicates) was used to evaluate the degree of support for a particular grouping pattern in the phylogenetic tree.

\section{Protein structure analysis}

The features of these putative MLO proteins (membrane spanning helices) were predicted using the online transmembrane helix prediction server, TMHMM, version 2.0 (www. cbs.dtu.dk/services/TMHMM/). The motifs were generated using MEME (http://meme.sdsc. edu/meme4_6_1/cgi-bin/meme.cgi) and visualized with Logo (http://weblogo.berkeley.edu/ logo.cgi). MEME was run from the web server with the minimum and maximum parameters of 6 and 50 amino acids, respectively, for each motif. The maximum number of motifs was 15 .

\section{Chromosomal locations of MLO proteins}

Information on the chromosomal location of $C s M L O$ genes in cucumber was collected from the Cucumber Genome Browser. Chromosomal locations were determined according to chromosomal information from the said database above.

\section{Expression profile investigation of $C S M L O$ genes}

To reveal the expression profiles of $C S M L O$ genes in different tissues, the Cucurbit Genomics Database (http://www.icugi.org/) was searched using the identified CsMLO genes. In this database, expressed sequence tag (EST) sequences of two species (cucumber and melon) were included. The nucleotide BLAST (BLASTn) program was used to search these EST sequences corresponding to the CsMLO genes.

\section{RESULTS}

\section{Identification of $C s M L O$ gene family members}

Homology searches of the published genome sequence of the cucumber against known MLO sequences from the plants mentioned above were performed to establish the number of $M L O$ genes in the cucumber 9930 genome. Fifteen genes were identified as possible members of the $M L O$ gene family. However, the nucleotide sequence of Csa026261 was identical to that of Csa014225. Therefore, the latter was excluded in the following analysis. Totally, $14 M L O$ genes were obtained from the cucumber 9930 genome and were named CsMLO01 through CsMLO14 (Table 1). Recently, a cucumber gynoecious inbred line (Gy14) was also sequenced de novo using an appropriate mixture of random shotgun and paired-end shotgun reads sequenced with 454-XLR technology (Cavagnaro et al., 2010). The extent of variability between cucumber GY14 and 9930 for $M L O$ genes was assessed using known MLO-like genes from 9930 and other plants in a BLAST search against the GY14 genome sequences (http://genome. 
jgi-psf.org/cucumber/cucumber.home.html). The same number of $M L O$ genes in cucumber GY14 was identified (Table S1). The comparison of 9930 and GY14 cucumber MLO genes showed that most of these genes are highly homologous ( $\underline{\text { Table S2 }}$ ).

\begin{tabular}{|c|c|c|c|c|c|c|c|c|}
\hline \multirow[t]{2}{*}{$\overline{\text { Gene }}$} & \multirow{2}{*}{$\begin{array}{l}\text { Cucumber } \\
\text { genomics ID }\end{array}$} & \multirow[t]{2}{*}{ Group } & \multirow[t]{2}{*}{$\mathrm{TM}^{\mathrm{b}}$} & \multirow[t]{2}{*}{ Genome position } & \multirow[t]{2}{*}{ ORF length (bp) } & \multicolumn{3}{|c|}{ Deduced polypeptide $^{\mathrm{c}}$} \\
\hline & & & & & & Length (aa) & Mol wt (kDa) & $\mathrm{pI}$ \\
\hline CsMLO01 & Csa026261 & II & 7 & Scaffold repeat 033789 & 1551 & 516 & 58.05 & 9.04 \\
\hline CsMLO02 02 & Csa000519 & III & 8 & Chr6: 23874952-23879410 & 1638 & 545 & 61.91 & 9.21 \\
\hline CsMLO03 & Csa009424 & I & 6 & Chr4: 20507811-20514326 & 1434 & 478 & 54.33 & 8.77 \\
\hline CsMLO04 & Csa010789 & I & 6 & Chr5: 26196794-26203304 & 1635 & 544 & 63.00 & 8.86 \\
\hline CsMLO05 & Csa010846 & $\mathrm{V}$ & 7 & Chr5: 25234662-25238627 & 1683 & 560 & 64.24 & 9.31 \\
\hline CsMLO06 & Csa013199 & III & 7 & Chr6: 14086212-14090951 & 1635 & 544 & 62.00 & 8.97 \\
\hline CsMLO07 & Csa015734 & I & 9 & Chr3: 785255-789423 & 1668 & 555 & 63.35 & 8.54 \\
\hline CsMLO08 & Csa015766 & III & 8 & Chr3: 1120867-1124790 & 1824 & 607 & 68.71 & 8.53 \\
\hline CsMLO09 1010 & Csa015858 & I & 5 & Chr3: 14311254-14323456 & 1842 & 613 & 70.11 & 8.82 \\
\hline CsMLO10 & Csa017196 & VI & 1 & Chr1: 8903233-8903945 & 513 & 170 & 19.16 & 6.15 \\
\hline CsMLO11 12 & Csa017197 & VII & 3 & Chr1: 8894761-8903171 & 1080 & 359 & 40.88 & 7.32 \\
\hline CsMLO12 13 & Csa017252 & II & 5 & Chr2: 13500696-13505621 & 1017 & 338 & 40.34 & 8.61 \\
\hline CsMLO13 1414 & Csa019070 & $\mathrm{V}$ & 7 & Chr1: 8275566-8281375 & 1749 & 582 & 66.91 & 9.21 \\
\hline CsMLO1415 & Csa020079 & $\mathrm{V}$ & 7 & Chr6: 12426669-12431612 & 1596 & 531 & 60.75 & 9.17 \\
\hline
\end{tabular}

${ }^{a}$ Available at [http://cucumber.genomics.org.cn/page/cucumber/index.jsp]. ${ }^{b}$ Number of transmembrance (TM) domains in the CSMLO proteins predicted by TMHMM transmembrance helix prediction software at the $80 \%$ probability level (www. cbs.dtu.dk/services/Tmhmm/). ${ }^{\mathrm{C}}$ Length (number of amino acids; aa), molecular weight (Mol wt; kiloDaltons), and isoelectric point ( $\mathrm{pI}$ ) of the deduced polypeptide.

Previous studies of the nucleotide-binding site/leucine-rich repeat (NBS-LRR) class of resistance genes from Arabidopsis and rice revealed that there are 149 and 480 resistant genes in these species, respectively (Meyers et al., 2003; Zhou et al., 2004). However, the number of $M L O$ genes in the 2 plant genomes was 15 and 12, respectively (Devoto et al., 2003; Liu and Zhu, 2008), which was less than that of the NBS-LRR class of resistant genes. In this report, $14 M L O$ genes, which were similar to those from Arabidopsis and rice, were identified in the cucumber. Therefore, we infer that $M L O$ genes are a relatively small family in plants compared with the NBS-LRR class of resistant genes.

The genomic location and predicted protein size for these $C s M L O$ genes were further identified (Table 1) based on the data provided by the Cucumber Genome Sequence Database (Huang et al., 2009). Moreover, the molecular weight (kDa) and isoelectric point of these CsMLO-deduced polypeptides were also calculated (Table 1). The predicted nucleotide and amino acid sequences of all 28 CsMLOs from 9930 and Gy14 are provided in Table S1.

\section{Structural features of CsMLO proteins}

Previous studies have experimentally uncovered MLO as an integral plasma membrane-resident protein with seven TM helices, an extracellularly located N-terminus, and a cytoplasmic C-terminus (Devoto et al., 1999). The TMs of the 14 CsMLO genes in this study were predicted with TMHMM. The proteins of these genes were found to have different numbers of TM domains, ranging from one (CsMLO10) to nine (CsMLO07) (Figure S1). Five CsMLO genes (CsMLO01, CsMLO05, CsMLO06, CsMLO13, and CsMLO14) were found to 
have 7 TMs (TM1 through TM7), which is characteristic of barley $M L O$ genes. However, sequence alignment further revealed that CsMLO05 lacked the TM2 domain and had an additional TM domain between the TM3 and TM4 domains. CsMLO14 lacked two TM domains (TM4 and TM5) and had two additional TM domains between the TM3 and TM6 domains. Three CsMLO genes (CsMLO02, CsMLO7, and CsMLO08) not only contained all seven TMs but also had an additional one, two, and one TM domains, respectively. In the four remaining CsMLO genes, the seven TMs presented various degrees of conservation (Figure 1A). The TM domain sequence logo was generated via an online logo tool (Crooks et al., 2004) (Figure 1B and Table 2) to highlight the amino acid residues conserved in the seven TM domains. Most of the amino acid residues in six of the TM domains were highly conserved; TM7 showed a degree of variation.

A

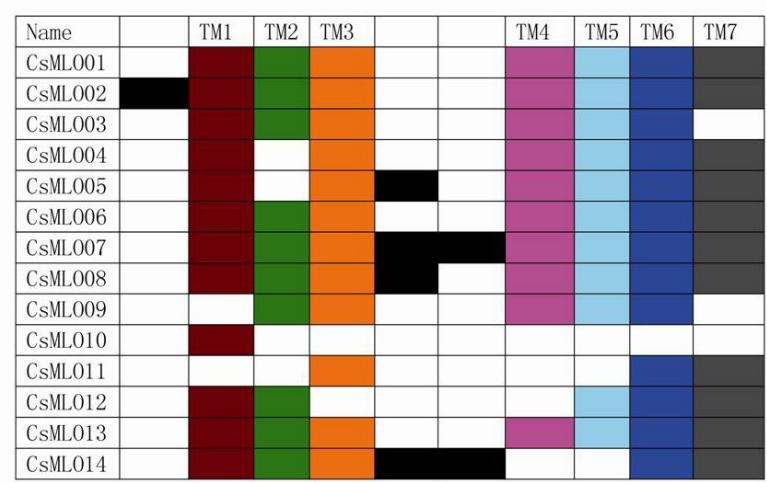

B
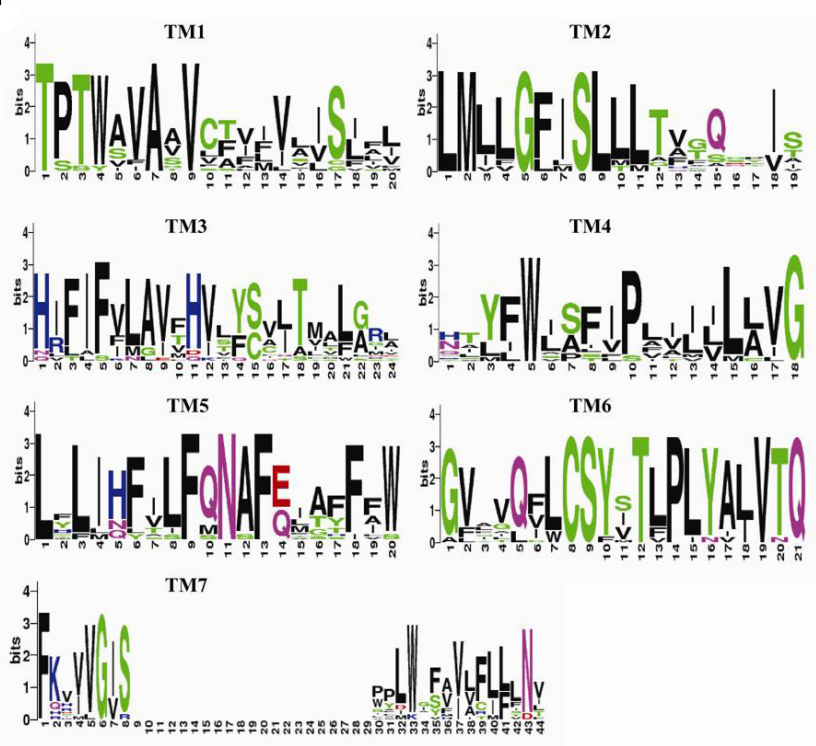

Figure 1. A. Number and distribution of transmembrance (TM) domains in the CsMLO proteins predicted by the TMHMM transmembrance helix prediction software (www. cbs.dtu.dk/services/Tmhmm/). B. Seven TM sequence $\log$ os in the CsMLO genes. 
Table 2. Major motifs in transmembrance domain within cucumber CsMLO genes.

\begin{tabular}{|c|c|c|}
\hline Domain & Motif & Sequence \\
\hline \multirow{7}{*}{ Transmembrance domain } & TM1 & $\underline{\text { TPTWAVA }}$ A/vVCT/fVIVL/aISI/LfT* \\
\hline & TM2 & L̄MLLGFISLLL̄TTVQxxIS \\
\hline & TM3 & 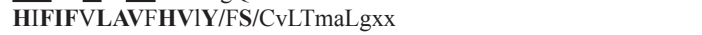 \\
\hline & TM4 & $\mathrm{HtYF} \underline{\mathbf{W}} / / \mathrm{SS} / \mathrm{aFIPli} / \mathrm{II} / \mathrm{LLL} / \mathrm{aV} \underline{\mathrm{G}}$ \\
\hline & TM5 & 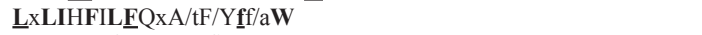 \\
\hline & TM6 & 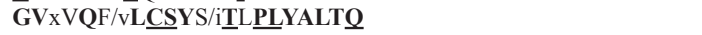 \\
\hline & TM7 & 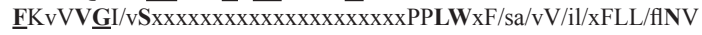 \\
\hline
\end{tabular}

*If the bits value (See Figure 1B) of the amino acid at this position is smaller that 0.5 , it is represented with $\mathrm{x} ; 1>$ bits $\geq 0.5$, with lowercase; $2>$ bits $\geq 1$, with capital letter; $3>$ bits $\geq 2$, with bold capital; bits $>3$, with underlined capital letter in bold.

\section{Sequence alignment and conserved motifs of CsMLO proteins}

Multiple sequence alignment of 14 CsMLO and barley MLO proteins revealed the presence of seven TM domains and 30 amino acid residues that have been previously identified as invariable in 38 MLOs from various species (Elliott et al., 2005) (Figure S2). Twelve of these amino acid residues were located in six of the seven TM domains. We further analyzed the conservation of these amino acid residues in each CsMLO. Six CsMLO proteins had all 30 amino acid residues conserved. Seven CsMLOs (CsMLO03, CsMLO04, CsMLO05, CsMLO10, CsMLO11, CsMLO12, and CsMLO14) lost one or more of the amino acid residues. Some amino acid residues of five CsMLOs (CsMLO05, CsMLO07, CsMLO10, CsMLO11, and CsMLO12) were mutated. However, most of the CsMLOs retained all four extracellular Cys residues (C86, C98, C114, and C367), which were demonstrated to be essential for the function of barley MLO (Elliott et al., 2005) (Table 3).

Subsequently, we used MEME to search for motifs in the CsMLOs. Seven of the 10 identified conserved motifs (Figure S3A) (motif 1 through motif 4 and motif 6 through motif 10) were located in the seven TM domains and CaMBD. The corresponding seven TMs were highly conserved. The remaining three motifs were located in other regions. Amino acid residues of each motif were highly conserved (Figure S3B).

\section{Phylogenetic relationship of $M L O$ genes in cucumber and other plants}

A phylogenetic tree containing $48 M L O$ genes from dicots and monocots was constructed to illustrate the phylogenetic relationships among the $M L O$ gene family members in cucumber and other plants. Seven distinct clades (I through VII) were identified (Figure 2). The designation of these clades was based on the classification by Devoto et al. (2003). Phylogenetic analysis showed that the $C s M L O$ genes are distributed in six of the seven clades. Clades IV and V included some $M L O$ genes from dicots and monocots that were previously identified to be involved in PM susceptibility (Jørgensen, 1992; Elliott et al., 2002; Kim et al., 2002b; Consonni et al., 2006). In clade IV, HvMLO, which causes PM susceptibility, is orthologous to the rice (OsMLO02) MLO proteins (Elliott et al., 2002). Moreover, this clade has been previously identified as a monocot-specific group (Devoto et al., 2003). In clade V, three CsMLOs (CsMLO05, CsMLO13, and CsMLO14) were clustered with AtMLO02, AtMLO06, and AtMLO12 from Arabidopsis, SIMLO from tomato, and PSMLO from pea. Additionally, amino acid sequences of the identified $M L O$ genes were aligned using the BioEdit software, 


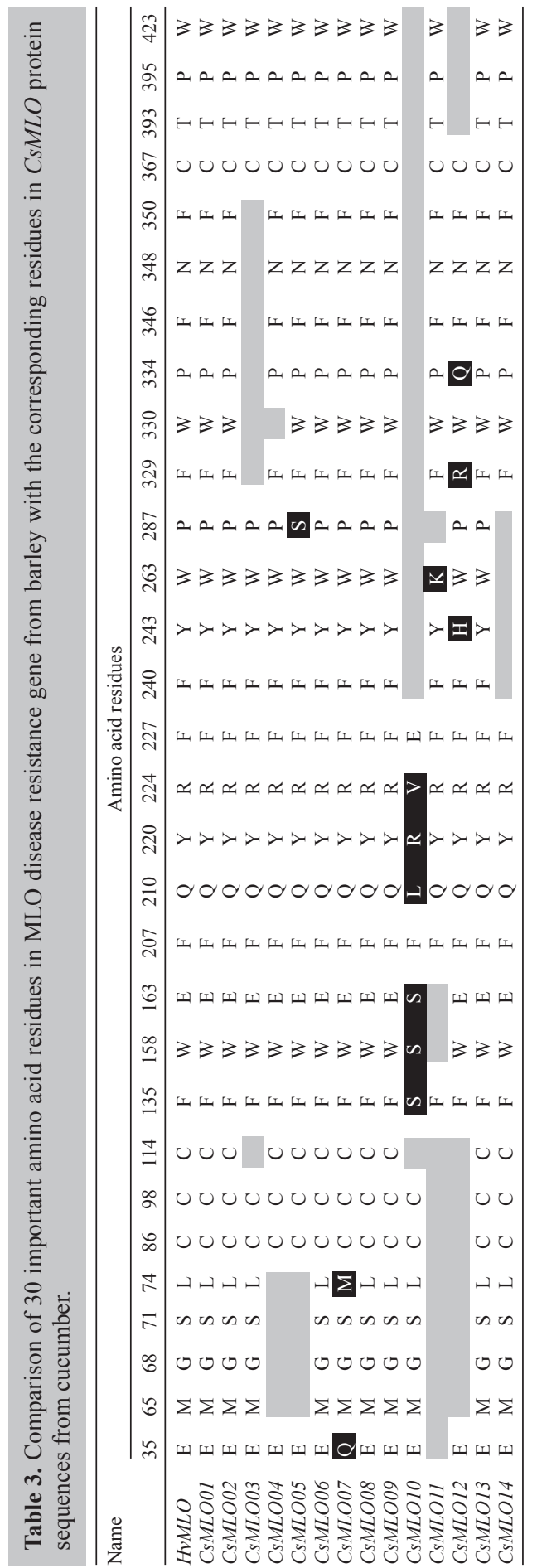


version 7.0.0 (Figure 3) to examine further the sequence conservation between the different genes from Arabidopsis, tomato, pea, and cucumber in clade V. In addition to the absence of the TM2 domain in CsMLO05, as well as the absence of the TM4 and TM5 domains in CsMLO14, all of the proteins showed a high degree of conservation at the position of the predicted TM domains (Devoto et al., 2003). Moreover, a CaMBD in the C-terminus of MLO proteins, which is conserved throughout the Arabidopsis MLO family (Kim et al., 2002a,b), was found to be highly conserved in 8 different members of the aforementioned plants that were analyzed. Additionally, in the C-terminus of MLO proteins, Panstruga (2005) also identified two other conserved regions (I and II) that play important roles in modulating PM infection. Peptide domain I is located approximately 15-20 residues downstream of the CaMBD and is characterized by conserved serine and threonine residues. Peptide domain II is located at the distal end of the C-terminus and contains the consensus sequence D/E-F-S/T-F. All eight MLOs had these two conserved domains within the C-terminus (Figure 3) in this study. The potential functional conservation of the MLO proteins that contain a CaMBD, as well as peptide domains I and II, is indicated in all of these findings.

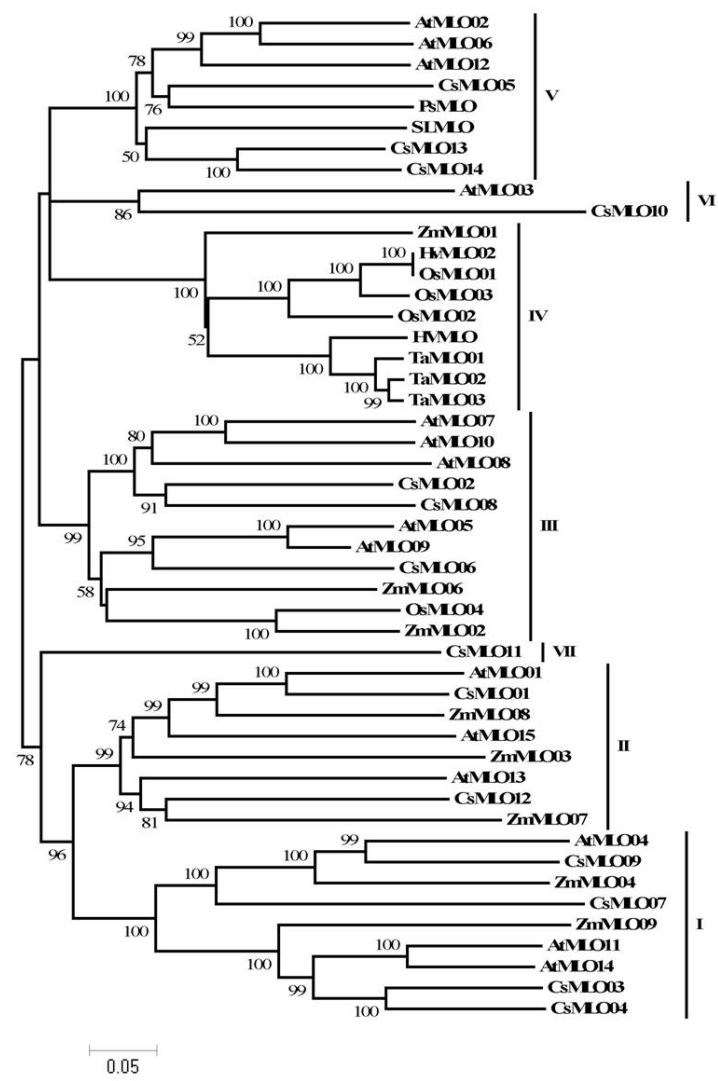

Figure 2. Phylogenetic relationship of $C s M L O$ in cucumber and $M L O$ s of other plant species including Arabidopsis $(A t M L O)$, tomato $(S I M L O)$, pea $(P S M L O)$, maize $(Z m M L O)$, barley $(H v M L O)$, rice $(O s M L O)$, and wheat $(T a M L O)$. The unrooted tree was generated using the MEGA 5.0 software and the neighbor-joining method. Bootstrap values (above 50\%) from 1000 replicates are indicated at each node. 


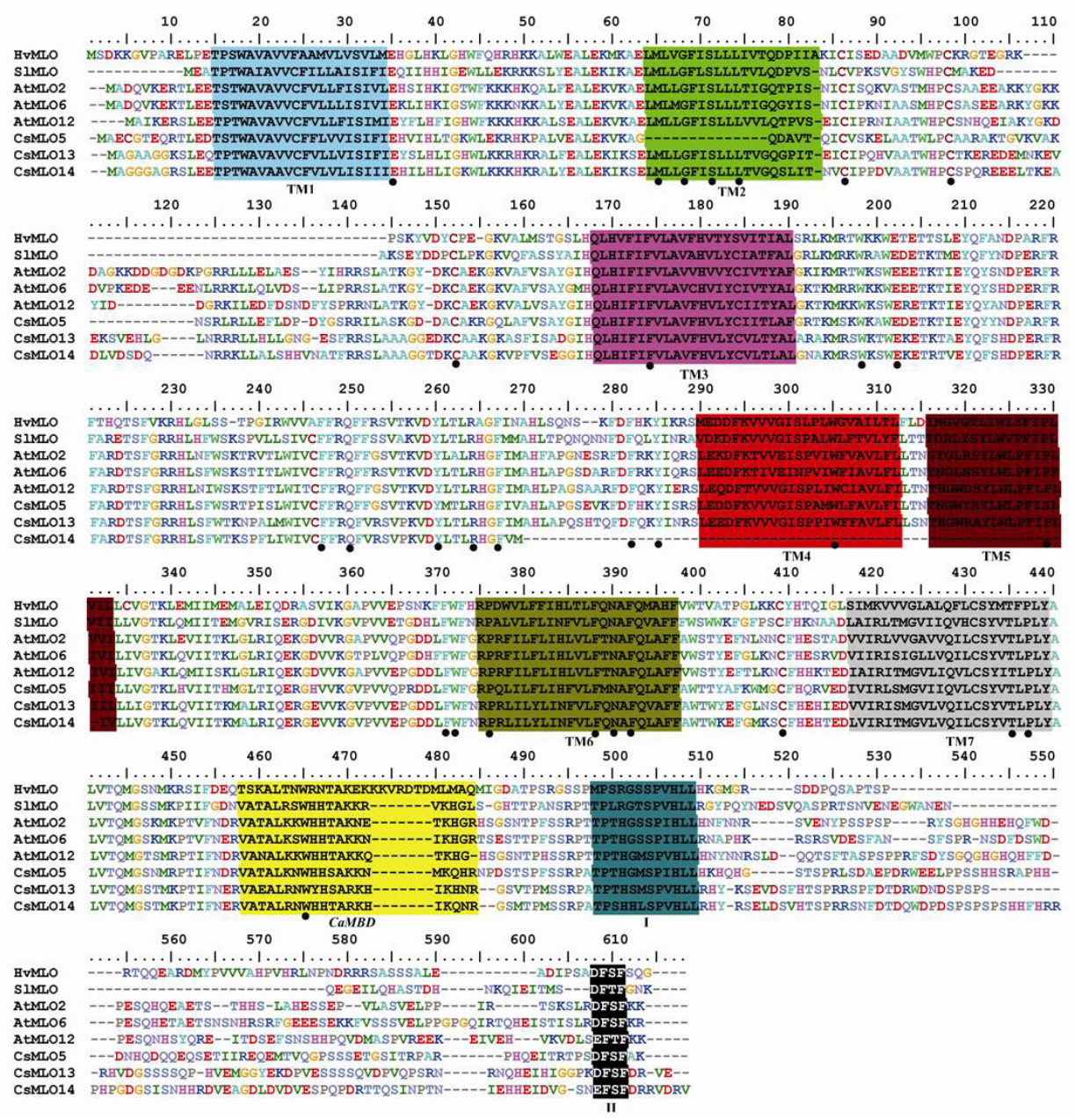

Figure 3. Multiple sequence alignment constructed using the Clustal $\mathrm{X}$ software of CsMLO genes in clade $\mathrm{V}$ (Figure 1) with selected MLO proteins involved in PM susceptibility in pea (PSMLO) (Pavan et al., 2011), tomato (SIMLO) (Bai et al., 2008), and Arabidopsis (AtMLO02, AtMLO06, and AtMLO12) (Consonni et al., 2006). The positions of the seven TM domains (TM1 to TM7) inferred from the experimentally determined topology of HvMLO (Devoto et al., 1999) and the approximate position of the CaMBD (Kim et al., 2002b) are indicated by lines under the sequences. Two conserved domains identified by Panstruga (2005) within the highly polymorphic C-termini are highlighted using Roman numerals I and II.

\section{Locations of the $M L O$ genes on the $C$. sativus chromosomes}

Fourteen $M L O$ family members have been identified in the cucumber genome. In silico mapping of the genes showed that $13 M L O$ genes were localized on six of seven chromosomes. The remaining $M L O$ gene, Csa026261 (CsMLO01), was assigned to the Scaffold_repeat 033789 . The distribution of $M L O$ family members in the cucumber genome is depicted in Figure 4. Two CsMLO genes, Csa009424 and Csa017252, were positioned on chromosomes 
4 and 2, respectively. Csa010846 and Csa010789 were both located on chromosome 5. Each of the remaining chromosomes included three $M L O$ genes. None of the $C s M L O$ genes were localized on chromosome 7. The cucumber $M L O$ genes showed a scattered distribution pattern on chromosomes.

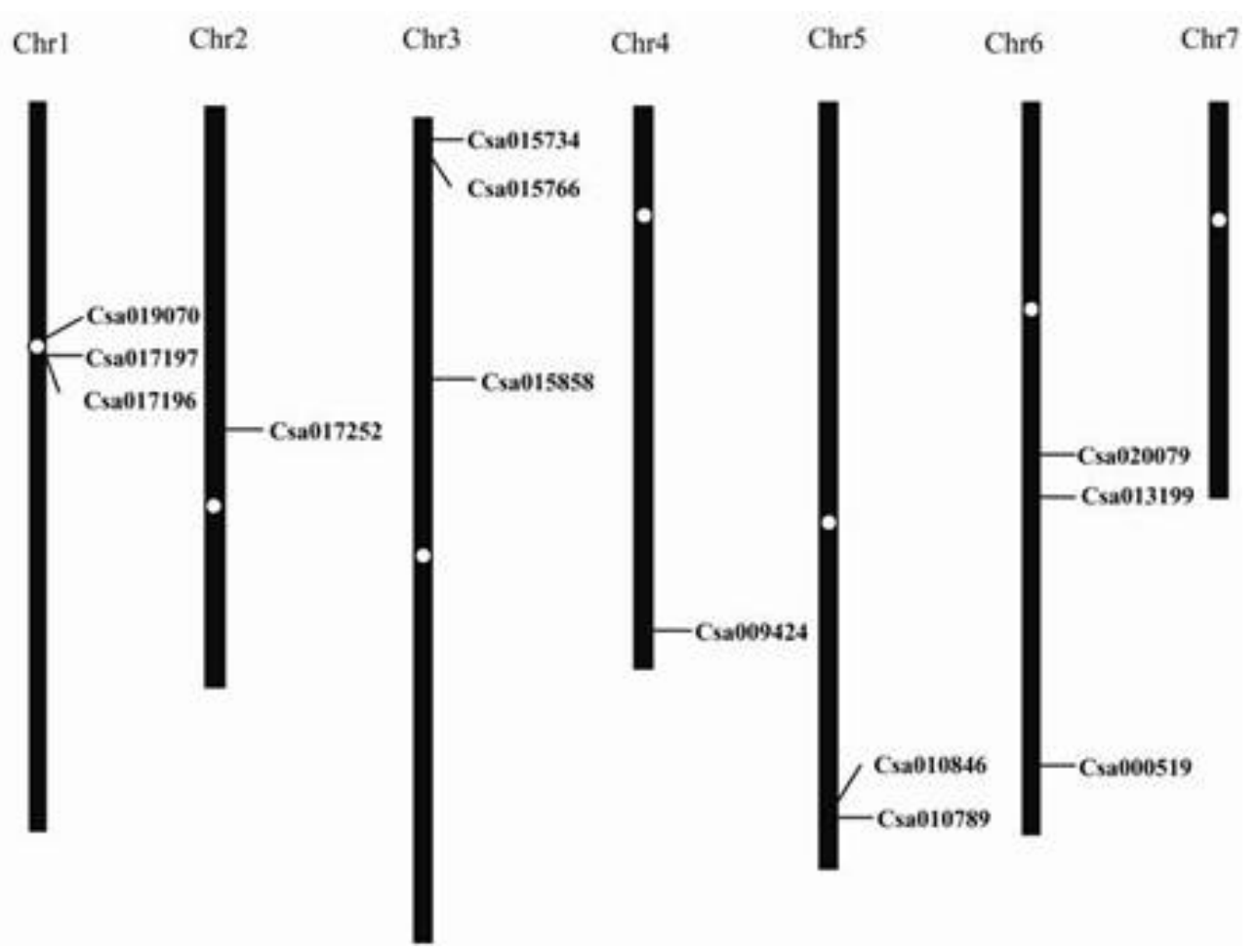

Figure 4. Positions of CsMLO genes on the cucumber chromosomes. Chromosome numbers are indicated on top of the chromosome.

\section{In silico analysis of CsMLO gene expression using EST libraries}

The mass of sequences available from different EST libraries can provide valuable information for gene expression analysis in plant research. In this paper, to assess which genes have expression data, the Cucurbit Genomics Database was searched with the coding region sequences of the CsMLO genes using the BLASTn program. The results showed that 10 and 12 CsMLO genes have EST data in cucumber and melon EST libraries, respectively (Table 4). The remaining $C s M L O$ genes were not detected in these two EST libraries. This indicated that these genes may have specific temporal and spatial expression patterns. In the cucumber EST library, a total of 129 EST matches were identified, with an average of 12.9 ESTs per expressed CsMLO gene. In the melon EST library, only 38 EST matches were obtained, with an average of 3.8 ESTs per expressed $M L O$ gene. 


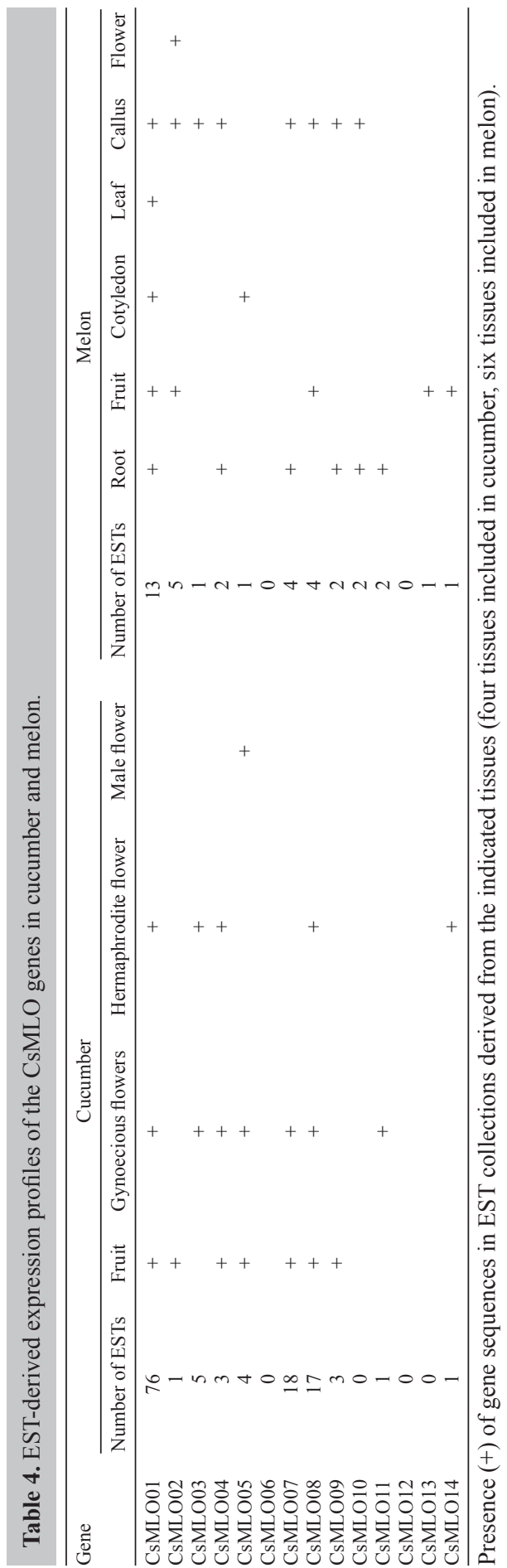




\section{DISCUSSION}

Sequence diversity of MLO family members in A. thaliana, their topology and subcellular localization are reminiscent of the G-protein-coupled receptor (GPCR) superfamily in metazoans (Devoto et al., 1999). The roles of GPCRs in metazoans involve the transfer of extracellular stimuli into intracellular signaling events by activating heterotrimeric G-proteins. Several human pathogens exploit host GPCRs for successful infection, including the human immunodeficiency virus type 1 and the bacterium Streptococcus pneumoniae (Pease and Murphy, 1998). These facts raise the question of whether MLO proteins in plants play a similar role during plant colonization by PM fungi.

Given that the putative functions of these $M L O \mathrm{~s}$ in clade $\mathrm{V}$ were derived from Arabidopsis, tomato, and pea, we infer that this clade is significant for cucumber because these genes are required for PM susceptibility (Consonni et al., 2006; Bai et al., 2008; Pavan et al., 2011). This clade may thus be a dicot-specific group. Clades IV and V contain the monocot and dicot $M L O \mathrm{~s}$, respectively, that were previously described to be involved in PM susceptibility (Jørgensen, 1992; Elliott et al., 2002; Kim et al., 2002b; Consonni et al., 2006). Therefore, we infer that $M L O$ genes associated with PM susceptibility in plants may have evolved after the divergence of monocots and dicots.

Previously, some studies have reported that several gene families could arise through tandem duplication of chromosomal regions, resulting in a clustered occurrence of family members, or through segmental duplication, resulting in a scattered occurrence of family members (Schauser et al., 2005). We assume that the expansion of the cucumber $M L O$ gene family mainly resulted from segmental duplications rather than tandem duplication.

The ESTs were identified for most CsMLO genes in two EST libraries, and most of them were expressed in fruit, root, and leaf (Table 4). This indicated that these $C s M L O$ genes may be involved in plant growth and development. Altogether, the large number of CsMLO genes with expression support provided very useful information for further experimental verification.

\section{Supplementary material}

\section{REFERENCES}

Bai Y, Pavan S, Zheng Z, Zappel NF, et al. (2008). Naturally occurring broad-spectrum powdery mildew resistance in a Central American tomato accession is caused by loss of mlo function. Mol. Plant Microbe Interact. 21: 30-39.

Bhat RA, Miklis M, Schmelzer E, Schulze-Lefert P, et al. (2005). Recruitment and interaction dynamics of plant penetration resistance components in a plasma membrane microdomain. Proc. Natl. Acad. Sci. U. S. A. 102: 3135-3140.

Buschges R, Hollricher K, Panstruga R, Simons G, et al. (1997). The barley Mlo gene: a novel control element of plant pathogen resistance. Cell 88: 695-705.

Cavagnaro PF, Senalik DA, Yang L, Simon PW, et al. (2010). Genome-wide characterization of simple sequence repeats in cucumber (Cucumis sativus L.). BMC Genomics 11: 569.

Consonni C, Humphry ME, Hartmann HA, Livaja M, et al. (2006). Conserved requirement for a plant host cell protein in powdery mildew pathogenesis. Nat. Genet. 38: 716-720.

Crooks GE, Hon G, Chandonia JM and Brenner SE (2004). WebLogo: a sequence logo generator. Genome Res. 14: 11881190.

Devoto A, Piffanelli P, Nilsson I, Wallin E, et al. (1999). Topology, subcellular localization, and sequence diversity of the Mlo family in plants. J. Biol. Chem. 274: 34993-35004.

Devoto A, Hartmann HA, Piffanelli P, Elliott C, et al. (2003). Molecular phylogeny and evolution of the plant-specific seven-transmembrane MLO family. J. Mol. Evol. 56: 77-88. 
Elliott C, Zhou F, Spielmeyer W, Panstruga R, et al. (2002). Functional conservation of wheat and rice Mlo orthologs in defense modulation to the powdery mildew fungus. Mol. Plant Microbe Interact. 15: 1069-1077.

Elliott C, Muller J, Miklis M, Bhat RA, et al. (2005). Conserved extracellular cysteine residues and cytoplasmic loop-loop interplay are required for functionality of the heptahelical MLO protein. Biochem. J. 385: 243-254.

Fanourakis NE (1984). Inheritance and Linkage Studies of the Fruit Epidermis Structure, and Investigation of Linkage Relations of Several Traits and of Meiosis in Cucumber. University of Wisconsin, Madison.

Fanourakis NE and Simon PW (1987). Analysis of genetic linkage in the cucumber. J. Hered. 78: 238-242.

Freialdenhoven A, Peterhansel C, Kurth J, Kreuzaler F, et al. (1996). Identification of genes required for the function of non-race-specific mlo resistance to powdery mildew in barley. Plant Cell 8: 5-14.

Huang S, Li R, Zhang Z, Li L, et al. (2009). The genome of the cucumber, Cucumis sativus L. Nat. Genet. 41: 1275-1281.

Jabs T, Tschope M, Colling C, Hahlbrock K, et al. (1997). Elicitor-stimulated ion fluxes and $\mathrm{O}_{2}^{-}$from the oxidative burst are essential components in triggering defense gene activation and phytoalexin synthesis in parsley. Proc. Natl. Acad. Sci. U. S. A. 94: 4800-4805.

Jørgensen IH (1992). Discovery, characterization and exploitation of Mlo powdery mildew resistance in barley. Euphytica 63: $141-152$

Kim MC, Lee SH, Kim JK, Chun HJ, et al. (2002a). Mlo, a modulator of plant defense and cell death, is a novel calmodulinbinding protein. Isolation and characterization of a rice Mlo homologue. J. Biol. Chem. 277: 19304-19314.

Kim MC, Panstruga R, Elliott C, Muller J, et al. (2002b). Calmodulin interacts with MLO protein to regulate defence against mildew in barley. Nature 416: 447-451.

Liu Q and Zhu H (2008). Molecular evolution of the MLO gene family in Oryza sativa and their functional divergence. Gene 409: 1-10.

Meyers BC, Kozik A, Griego A, Kuang H, et al. (2003). Genome-wide analysis of NBS-LRR-encoding genes in Arabidopsis. Plant Cell 15: 809-834.

Panstruga R (2005). Serpentine plant MLO proteins as entry portals for powdery mildew fungi. Biochem. Soc. Trans. 33: 389-392.

Pavan S, Schiavulli A, Appiano M, Marcotrigiano AR, et al. (2011). Pea powdery mildew er1 resistance is associated to loss-of-function mutations at a MLO homologous locus. Theor. Appl. Genet. 123: 1425-1431.

Pease JE and Murphy PM (1998). Microbial corruption of the chemokine system: an expanding paradigm. Semin. Immunol. 10: 169-178.

Peterhansel C, Freialdenhoven A, Kurth J, Kolsch R, et al. (1997). Interaction analyses of genes required for resistance responses to powdery mildew in barley reveal distinct pathways leading to leaf cell death. Plant Cell 9: 1397-1409.

Pierce LK and Wehner TC (1990). Review of genes and linkage groups in cucumber. Hort. Sci. 25: 605-615.

Piffanelli P, Ramsay L, Waugh R, Benabdelmouna A, et al. (2004). A barley cultivation-associated polymorphism conveys resistance to powdery mildew. Nature 430: 887-891.

Pivovarov V (1988). Cucumber Breeding for General Adaptive Ability and Resistance to Diseases [Pseudoperonospora cubensis, Sphaerotheca fuliginea, Erysiphe cichoracearum]. INRA, Paris.

Sakata Y, Kubo N, Morishita M, Kitadani E, et al. (2006). QTL analysis of powdery mildew resistance in cucumber (Cucumis sativus L.). Theor. Appl. Genet. 112: 243-250.

Schauser L, Wieloch W and Stougaard J (2005). Evolution of NIN-like proteins in Arabidopsis, rice, and Lotus japonicus. J. Mol. Evol. 60: 229-237.

Schultheiss H, Dechert C, Kogel KH and Huckelhoven R (2002). A small GTP-binding host protein is required for entry of powdery mildew fungus into epidermal cells of barley. Plant Physiol. 128: 1447-1454.

Tamura K, Peterson D, Peterson N, Stecher G, et al. (2011). MEGA5: molecular evolutionary genetics analysis using maximum likelihood, evolutionary distance, and maximum parsimony methods. Mol. Biol. Evol. 28: 2731-2739.

Thompson JD, Gibson TJ, Plewniak F, Jeanmougin F, et al. (1997). The CLUSTAL_X windows interface: flexible strategies for multiple sequence alignment aided by quality analysis tools. Nucleic Acids Res. 25: 4876-4882.

Van Vliet GJA and Meijsing WD (1977). Relation in the inheritance of resistance to Pseudoperonospora cubensis rost and Sphaerotheca fuliginea Poll. in cucumber (Cucumis sativus L.). Euphytica 26: 793-796.

Walters SA, Shetty NV and Wehner TC (2001). Segregation and linkage of several genes in cucumber. J. Am. Soc. Hort. Sci. 126: 442-450.

$\mathrm{Xu} \mathrm{H}$ and Heath MC (1998). Role of calcium in signal transduction during the hypersensitive response caused by basidiospore-derived infection of the cowpea rust fungus. Plant Cell 10: 585-598.

Zhang SQ, Gu XF, Zhang SP and Zou ZR (2007). Inheritance of powdery mildew resistance in cucumber (Cucumis sativus L.) and development of an AFLP marker for resistance detection. Agric. Sci. China 6: 1336-1342.

Zhou T, Wang Y, Chen JQ, Araki H, et al. (2004). Genome-wide identification of NBS genes in japonica rice reveals significant expansion of divergent non-TIR NBS-LRR genes. Mol. Genet. Genomics 271: 402-415. 